\title{
The Impact of the Third Wave of COVID 19 in Patients with Chronic Hepatitis $\mathbf{C}$
}

Daniela Mariano Carvalho-Louro*

Gastroenterology Unit, Instituto Hospital de Base, Brazil

\section{Letter to Editor}

In the practical clinical of Hepatology, the focus of daily life has been the treatment of patients with severe liver diseases, such as cirrhosis and liver cancer. Of all the liver diseases responsible for cirrhosis development, hepatitis $\mathrm{C}$ had made the most treatment progress. In a few years, it evolved from drugs with low efficacy and many side effects to highly safe medications with high cure rates.

For hepatologists who manage critically ill patients with advanced stages of liver diseases, finding an effective Hepatitis $\mathrm{C}$ drug was a great encouragement, a huge motivation to continue believing in clinical research.

World Health Organization (WHO) estimated 1\% of the world population is chronic carriers of Hepatitis C. Since the emergence of these new drugs, the challenge has been to find patients with Hepatitis $\mathrm{C}$ before cirrhosis and liver cancer.

The WHO initiated, in 2017, a strategic plan to eliminate viral Hepatitis by 2030. In hepatitis $C$, the predicted goal was a $90 \%$ reduction in the rate of new infections with a $65 \%$ decrease in mortality from disease. The nations have been on a mission to increase individuals' testing and facilitate chronic patients' access to a specific treatment. Global efforts are crucial to improving patients' access to treatment. However, in 2020, a new threat - COVID 19, started to haunt the population, causing stop progress on the project to eliminating Hepatitis C.

We were experiencing the peak of successful treatments in previous years, and the hope of stopping new cirrhosis and liver cancer cases caused by Hepatitis C, and liver cancer cases caused by Hepatitis $\mathrm{C}$, the leading cause of liver the leading cause of liver transplantation worldwide. However, during the year 2020, the number of new diagnoses of chronic carriers and new treatments plummeted in many countries. The last months were permeated by the discussions of treatment and return to activities during the pandemic of the COVID-19.

Many questions have been appearing during the pandemic period, ones with obvious answers, but others with solutions still under development: Did chronic diseases, previously existing, disappear during the pandemic? Where are the Hepatitis $\mathrm{C}$ patients who are unaware of their diagnosis at this time of epidemic? Do chronic patients remain socially distant, and their liver disease evolves, with an imminent risk of cirrhosis or even liver cancer?

The pandemic has several impacts on public health and these impacts are called waves. The effect of assistance lack of people with chronic diseases during the epidemic are called the third wave. It will increase the number of critically ill patients and, again, in the case of Hepatitis $\mathrm{C}$ with decompensation of cirrhosis and development of liver cancer, overloading the health systems that, despite many difficulties have faced bravely the COVID 19.

Despite all efforts until today and effective hepatitis $C$ therapies, the chronic patients will arrive at the specialist with advanced liver disease stages in the next years. It is up to doctors and health managers to reflect that life does not stop during a pandemic. Hepatitis $C$ is a real fact, but hypertension, AIDS, diabetes, and other infectious diseases are examples of the many health problems suffered by the population, mainly in underdeveloped countries, just as crucial as COVID.

The health of chronic patients cannot wait for better times. Many diseases affect the population and require immediate actions to provide them access, diagnosis, and treatment, minimizing the third wave's effect, avoiding the worsening of the overload situation the all healthy systems are experiencing today.

Citation: Carvalho-Louro DM. The Impact of the Third Wave of COVID 19 in Patients with Chronic Hepatitis C. J Clin Pharmacol Ther 2020;1(1):1003.

Copyright: $\odot 2020$ Carvalho-Louro DM

Publisher Name: Medtext Publications LLC

Manuscript compiled: Oct $28^{\text {th }}, 2020$

*Corresponding author: Carvalho-Louro DM, Gastroenterology Unit, Instituto Hospital de Base, Brasilia, Federal District, 70322-000, Brazil, E-mail: daniela.mac@gmail.com 